\title{
Veröffentlichungen \\ von Prof. Dr. med. Huldrych M. Koelbing
}

Nachtrag und Fortsetzung der in Gesnerus 40 (1983) erschienen Bibliographie

\section{Monographien und Editionen}

Bericht über einen Fortbildungskurs in industrieller Ophthalmologie, veranstaltet vom Research Departement des Birmingham and Midland Eye Hospital, 1954. Typoskript 1954 (unveröff.?), $32 \mathrm{~S}$.

Erwin H.Ackerknecht: Medicine and ethnology. Selected essays. Ed. by H.H. Walser and H. M. Koelbing. Bern 1971, 195 S. - Trad. castellana ampliada, Madrid 1985, 218 S.

Die ärztliche Therapie. Grundzüge ihrer Geschichte. Darmstadt 1985, 271 S. - Edizione italiana, Saronno 1989, $221 \mathrm{~S}$.

Johann Friedrich Horner, 1831-1886. Der Begründer der Schweizer Augenheilkunde in seiner Autobiographie. Nach dem Manuskript neu hrsg. von Huldrych M.Koelbing und Christoph Mörgeli. Zürich 1986, 113 S.

\section{Aufsätze in Zeitschriften und Büchern}

Pockennarben und Schönheit. Gesnerus 37 (1980), S. 321-323.

Medizin seit 1945. Zeitgeschichte III : 1945-1980, 2. Teil, Prof. Dr. R. von Albertini, Vorlesung Universität Zürich Wintersemester 1982/83, S.77-82.

L'ophtalmologie en Suisse au XIXe siècle. L'ophtalmologie des origines à nos jours, t.4, Paris 1983, S.41-46.

Das Problem der visuellen Wahrnehmung in der Antike, im Mittelalter und in der Renaissance. Fortschr. der Ophthalmologie 80 (1983), S.11-14.

Lehren aus der Therapiegeschichte - der therapeutische Optimismus und seine Tücken. Schweiz. Med.Wschr. 113 (1983), S. 1378-1384. - Ebenfalls : internist. prax. 29 (1989), S.141149. - Teilabdruck: Sigmund Freud und das Kokain. euromed 12 (1983), S. 639-640.

Erklären, schweigen, täuschen? Beispiele zu einem Hauptaspekt der Arzt-Patient-Beziehung. Schweiz.Rundschau Med. (Praxis) 72 (1983), S. 1605-1606.

Bemerkungen zu Ingrid Rohlands und Gundolf Keils «Randnotizen zum «Schüpfheimer Kodexı». Gesnerus 40 (1983), S. 275-276.

Paracelsus und seine «Grosse Wundartzney» (1536/37). Schweiz. Ärztezeitung 65 (1984), S.21-25. (Mit R. Häusermann und K. Biener:) Präventivmedizin in den alten Religionen. Sozial- und Präventivmedizin 29 (1984), S. 72-77. 
Medizin und Ärzte vor 1834. Medizin und Ärzte im Glarnerland, hrsg. von der Ärzteges. des Kantons Glarus. Glarus 1984, S. 9-19.

Bekannte Glarner Ärzte: Pioniere der Operationsnarkose. Medizin und Ärzte im Glarnerland, hrsg. von der Ärzteges. des Kantons Glarus. Glarus 1984, S. 97-102.

Glarner Landärzte als Narkose-Pioniere. Swiss med.6 (1984), S. 13.

Platon und die hippokratische ärztliche Kunst. Neue Zürcher Zeitung, Nr. 162, S.45, 1984.

Hippokratische Medizin. Ein internationales Kolloquium in Berlin. Neue Zürcher Zeitung, Nr. 235, S. 39, 1984. Ebenfalls: Gesnerus 42 (1985), S. 161-165.

Zur Geschichte der Pest in der Schweiz. Jahrbuch für Solothurnische Geschichte, 57. Band, 1984, S.5-12.

Fabricius Hildanus (1560-1634), führender Chirurg und Gegner der Folter. Schweiz. Rundschau Med. (Praxis) 73 (1984), S.1565-1567.

Zur Erinnerung an Heinrich Buess, 1911-1984. Neue Zürcher Zeitung, Nr. 6, S. 31, 1985.

Leber, Theodor, Augenarzt. Neue deutsche Biographie, Bd.14, 1985, S.19-20.

Kühnheit und Umsicht: Jacques Daviels Weg zur Star-Extraktion (1745-1752). Klin.Monatsbl. für Augenheilkunde 136 (1985), S.235-238.

Mailand-Basel-Strassburg-Frankfurt. Informationsaustausch im Dienste der Pestabwehr im 17. Jahrhundert. «Istorgia dalla Madaschegna», Festschrift für Nikolaus Mani. Pattensen/Han. 1985, S. 157-169.

(Mit Luzius von Rechenberg:) Hufelands Opiumtherapie im zeitgenössischen Vergleich. Gesnerus 42 (1985), S. 97-119.

Lehren aus der Geschichte der Therapie. Über den therapeutischen Optimismus, seine Berechtigung und seine Gefahren. Probleme und Behandlungsfragen des alternden Kriegsbeschädigten, zus. gestellt von H. Hasse. Bonn 1985, S. 18-26. (Schriftenreihe des Bundesversorgungsblattes, Heft 15).

Zwischenstaatliche Pestabwehr vor 400 Jahren. Der Vertrag von Bellinzona (12. August 1585). Neue Zürcher Zeitung, Nr. 184, S. 17, 1985.

Zur Erinnerung an Heinrich Buess, 17.5.1911-31.12.1984. Gesnerus 42 (1985), S. 3-5.

Tierversuche spielten in der Medizin eine wichtige Rolle. Tages-Anzeiger, 13. Nov. 1985, S. 58.

Remarques à propos de l'évolution des connaissances sur le cerveau. Gesnerus 42 (1985), S. 315328.

Francis Bacon über Lebensverlängerung und Euthanasie. Schweiz. Rundschau Med. (Praxis) 74 (1985), S. 1441-1442.

Zur Befreiung des Konzentrationslagers Buchenwald. Ein Dokument. Schweiz.Zeitschr. f. Geschichte 35 (1985), S. 449-454.

La médecine vue dans une perspective historique. Revue CIC (Club International de Coopération) 1 (1985), S. 153-154.

Montpellier vu par Felix Platter, étudiant en médecine de 1552 à 1557. Histoire de l'Ecole médicale de Montpellier, Actes du $110^{\mathrm{e}}$ Congrès national des Sociétés savantes (Montpellier, 1985). Paris 1985, S. 85-94. 
Pestsperre am Gotthard. Der mailändisch-schweizerische Vertrag von 1585 im Lichte neuerer Forschungen. Schweiz.Rundschau Med. (Praxis) 75 (1986), S. 228-231.

Zum 80. Geburtstag von Erwin H. Ackerknecht, 1. Juni 1986. Schweiz.Rundschau Med. (Praxis) 75 (1986), S. 742.

Beziehungen zwischen der Schweiz und Deutschland im konfessionellen Zeitalter : der medizinische Bereich. Das Reich und die Eidgenossenschaft 1580-1650, hrsg. von Ulrich Im Hof und Suzanne Stehelin. Freiburg/Schweiz 1986, S. 239-257.

Wichtige Schritte der praktischen Medizin in 75 Jahren. Schweiz.Rundschau Med. (Praxis) 75 (1986), S. 1315-1322.

Kokainsucht schon vor 100 Jahren. A. Erlenmeyer: Über Cocainsucht - vorläufige Mitteilung. Deutsche Medizinal-Zeitung, 31. Mai 1886 (Nr.44), 483 f. Schweiz.Rundschau Med. (Praxis) 75 (1986), S.1565-1568.

(Mit A. Bollinger und H.Kupferschmidt:) Zur Geschichte der Mikrozirkulation aus klinischer Sicht. VASA 15 (1986), S. 335-339.

A Century of Psychiatry. Psychopathology 19 (1986), supp. 2, S.1-5.

Durch Krankheit an den Rand gedrängt: Die Aussätzigen im Mittelalter. unizürich 1/2, 1987, S. 10-12.

(Mit Vera Koelbing-Waldis:) Katastrophe und Herausforderung: Pest und Pestbekämpfung in Oberitalien und der Schweiz. Jahrbuch des Instituts für Geschichte der Medizin der Robert Bosch Stiftung 4 (1985). Stuttgart 1987, S. 7-21.

Zur Erinnerung an Erna Lesky (22. Mai 1911-17. November 1986). Gesnerus 44 (1987), S. 3-5.

Between Alhazen and Kepler: the treatise "On Sight" by Fabricius ab Aquapendente, Venice 1600. Advances in Diagnostic Visual Optics, Proceedings of the 3rd International Symposium, Tirrenia, Italy, 1986. Berlin 1987, S.11-14.

Die Folgen der Entdeckung eines Krankheitserregers am Beispiel von Lepra, Tuberkulose und Lues. Documenta Ciba-Geigy 6/87 (1987), S. 14-15.

Il trattato «De Visione» di Girolamo Fabrici d'Acquapendente (Venezia, 1600). Atti del XXXII Congresso Nazionale della Società Italiana di Storia della Medicina, Padova/Trieste 1985. Padova 1987, S. 29-33.

In Memoriam Erna Lesky (1911-1986). Bull. Hist. Med. 61 (1987), S.442-443.

Die Pest als historische Realität. Der Pestpatron Rochus, Roque, Rocco, Roch. Die Sammlung Engeler, hrsg. von Christoph Mörgeli. Zürich 1987, S. 5-7.

Das Arztgeheimnis in historischer Perspektive. USZ Nachrichten 3/1988, S.4-7.

Newton's and Goethe's colour theories - contradictory or complementary approaches? Theory and Experiment, D. Batens and J. P.van Bendegem (eds.). Dordrecht 1988, S. 189-206.

Seuchen und Seuchenbekämpfung im Mittelalter. Mensch, Medizin, Gesellschaft, hrsg. von Karl Hinteregger. Innsbruck 1988, S. 19-29.

(Ohne Unterschrift:) Die Zürcher Universität konnte gleich bei ihrer Eröffnung ... den medizinischen Unterricht mit fünf Lehrstühlen aufnehmen. Ein Rundgang im alten Hochschulquartier, hrsg. vom Prorektorat Lehre und Forschung der Universität Zürich, 1988, S. 16-17.

Zur Geschichte der Farbenlehre. Klin. Monatsblätter für Augenheilkunde 192 (1988), S.176-182. 
Weht der Geist, wo er will? Voraussetzungen medizinischer Entdeckungen. Schweiz. Med. Wschr. 118 (1988), S.1155-1162.

Von den fahrenden Starstechern zur ambulanten Augenchirurgie amerikanischen Stils. Schweiz. Ärztezeitung 69 (1988), S. 2013-2014.

Ärztliche Ethik im Lauf der Geschichte. Künstliches Leben - ärztliche Kunst?, hrsg. von Jürg von Ins und Peter Grossmann. Zürich 1989, S. 53-65. (Zürcher Hochschulforum, Band 12).

Professor Dr. Hanspeter Hartmann, 1. Mai 1929 bis 19. März 1988. Jahresbericht 1988/89 der Universität Zürich, S. 129-130.

Le médecin dans la cité grecque. Gesnerus 46 (1989), S.29-43.

Felix Bloch, 1919-1989. Gesnerus 46 (1989), S.117-118.

Hans R. Sauerländer, 1909-1989. Gesnerus 46 (1989), S. 118-119.

Eduard Seidler 60. Gesnerus 46 (1989), S. 120.

Therapeutischer Optimismus und therapeutische Zurückhaltung in der hippokratischen Medizin. Sudhoffs Archiv, Beiheft 27. Stuttgart 1989, S. 338-346.

Universität Zürich : Neuer Ordinarius für Geschichte der Medizin. Gesnerus 46 (1989), S. 271272.

Universität Wien: Neuer Vorsteher des Instituts für Geschichte der Medizin. Gesnerus 46 (1989), S. 281.

Johann Lucas Schönlein (1793-1864), Forscher und Kliniker. Der Hautarzt 41 (1990), S.174177.

Felix Platter (1536-1614) als Augenarzt. Gesnerus 47 (1990), S. 21-30.

(Mit Peter Speiser:) Der Graefe-Stein - eine Erinnerung an A. von Graefes Wirken in Heiden. Gesnerus 47 (1990), 109-117.

Georg Harig, 1935-1989. Gesnerus 47 (1990), S. 185-186.

(Mit Moritz Leisibach:) Zwischen Handwerk und Wissenschaft - das Medizinisch-Chirurgische Institut in Zürich, 1782-1833. Chirurgische Ausbildung im 18. Jahrhundert, hrsg. von Georg Harig. Husum 1990, S. 127-136. (Abhandlungen zur Geschichte der Medizin und der Naturwissenschaften, Heft 57).

Anatomie de l'œil et perception visuelle, de Vésale à Kepler. Le Corps à la Renaissance, Actes du XXX' Colloque de Tours 1987. Paris 1990, S. 389-397.

Un'impronta millenaria. L. Sterpellone : Stratigrafia di un passato. Milano 1990, S.44-45.

Un étudiant bâlois: Félix Platter. La Médecine à Montpellier du XII ${ }^{e}$ au XX $X^{e}$ siècle, sous la direction de Louis Dulieu. Paris 1990, S. 72-75.

Münsterlingen - das Akutspital und seine Entwicklung. 150 Jahre Münsterlingen. Das Thurgauische Kantonsspital und die Psychiatrische Klinik 1840-1990, hrsg. von Jürg Ammann und Karl Studer. Münsterlingen 1990, S.61-72.

Anatomia dell'occhio e percezione visiva nell'opera di G. Fabrizi d'Acquapendente. Acta Medicae Historiae Patavina XXXV e XXXVI, Anni Accademici 1988-1989 e 1989-1990, S.29-38.

Felix Platters Patienten. Ihre Krankheiten, Lebensverhältnisse und Schicksale im Spiegel der «Observationes». Felix Platter (1536-1614) in seiner Zeit, hrsg. von Ulrich Tröhler. Basel 1991, S. 60-67. (Basler Veröffentlichungen zur Geschichte der Medizin und der Biologie, N. F., Fasc. 3). 
Zur Sozialgeschichte der Medizin. Gesnerus 48 (1991), S. 103-107. (Essay review).

Heimsuchungen: Prüfung, Gnade und Herausforderung für den mittelalterlichen Menschen. Schweizer Hotel-Journal 21 (1991), S. 37-38.

Zwölfhundert Jahre griechische Medizin - im Altertum entstand die Grundlage einer Wissenschaft und eines Berufsethos: «Der Kranke muss sich mit dem Arzt gegen die Krankheit wehren». Der Landbote, Nr.212, S. 27, 1991.

Julius Hirschberg (1843-1925), oculiste actif et historien de l'ophtalmologie. 33 Beiträge zur Geschichte der Augenheilkunde, Julius Hirschberg Gesellschaft. Wien 1991, S.42-47.

Thomas Percival (1740-1804). Klassiker der Medizin, hrsg. von Dietrich v. Engelhardt und Fritz Hartmann. München 1991, Band 1, S. 276-290.

Joseph Lister (1827-1912). Klassiker der Medizin, hrsg. von Dietrich v. Engelhardt und Fritz Hartmann. München 1991, Band 2, S. 234-246.

Der Codex Schürstab um 1469: ein iatromathematisches Hausbuch. Zentralbibliothek Zürich: Schätze aus vierzehn Jahrhunderten, hrsg. von Alfred Cattani und Hans Jakob Haag. Zürich 1991, S.22-25.

Frontières confessionnelles et échanges culturels: le cas de la Suisse au XVI ${ }^{\mathrm{e}}$ siècle. Les frontières religieuses en Europe du $X V^{e}$ au $X V I I^{e}$ siècle, Actes du XXXI ${ }^{e}$ Colloque international d'études humanistes. Etudes réunies par Robert Sauzet. Paris 1992, S.195-205). (De Pétrarque à Descartes, LV).

Zum Columbus-Zentenarium 1492-1992. Gesnerus 49 (1992), S. 7-9.

Friedrich Rintelen †. Gesnerus 49 (1992), S. 63.

Zum Andenken an Friedrich Rintelen, 9. August 1906-3. November 1991. Mitteilungsblatt der Julius Hirschberg-Gesellschaft I/1992, S. 31.

Médecins, chirurgiens et livres entre Réforme et Contre-Réforme. Revue médicale de la Suisse Romande 112 (1992), S. 1045-1050.

\section{Buchbesprechungen}

Seit 1953 können wir rund 115 Rezensionen nachweisen, die in renommierten Tageszeitungen, in der medizinischen Fachpresse und zu einem grossen Teil auch in Gesnerus erschienen sind.

Zusammengestellt nach einer in der Bibliothek des Medizinhistorischen Instituts und Museums der Universität Zürich geführten Liste.

\section{L'Institut Louis Jeantet d'histoire de la médecine (Université de Genève): perspectives et projets}

Entretien avec le professeur Bernardino Fantini

Propos recueillis par Philippe Mudry 
Dans sa dernière livraison (49, 3/4, 1992, p. 389), Gesnerus a rendu compte sous la plume de Jean Jacques Dreifuss de la récente nomination de Bernardino Fantini à la tête de l'Institut Louis Jeantet d'histoire de la médecine (Université de Genève). Ce texte retraçait brièvement la carrière scientifique du nouveau professeur. Nous proposons aujourd'hui au lecteur un entretien avec le professeur Fantini à qui incombe désormais la tâche de diriger et d'animer l'institut genevois.

(Ph.M.) L'Institut Louis Jeantet d'histoire de la médecine est une création récente puisqu'il a été fondé en 1987. Pourriez-vous nous indiquer brièvement les conditions matérielles de son fonctionnement ainsi que les ressources humaines dont il dispose?

(B. F.) Il importe d'abord de préciser que l'Institut Louis Jeantet d'histoire de la médecine est un institut universitaire rattaché à la Faculté de médecine. Au moment de la création de cet institut, la Fondation Louis Jeantet a voulu contribuer à développer dans la Faculté de médecine un enseignement et un domaine de recherche qui n'en étaient certes pas absents: il suffit en effet de penser à l'enseignement qui a été donné par le professeur Jean Starobinski. Mais la chaire du professeur Starobinski était rattachée à la Faculté des lettres et non à la Faculté de médecine, qui ne disposait en ce qui concerne l'histoire de la médecine que d'un poste de privat docent. La Faculté de médecine a donc décidé la création d'un poste de professeur d'histoire de la médecine et a ouvert un concours pour le pourvoir. J'ai été nommé l'été dernier.

En ce qui concerne ses crédits de fonctionnement et les salaires, l'Institut dépend à la fois de la Fondation Louis Jeantet de médecine et de la Faculté de médecine. Sans entrer dans le détail, actuellement la proportion est à peu près de $50 \%$ pour chacune des parties. L'Institut dispose d'un budget suffisant pour lui assurer un fonctionnement que je qualifierai de normal, c'est-à-dire pour assumer ses tâches institutionnelles dans le domaine de l'enseignement et de la recherche. Mais contrairement à certaines idées reçues, le lien avec la Fondation Jeantet n'assure pas à l'Institut un pactole budgétaire. La mise sur pied de projets spéciaux et de plus grande envergure suppose que l'Institut trouve ailleurs des sources de financement complémentaires.

En ce qui concerne les locaux, l'Institut est installé dans la villa Thury, à proximité immédiate de la Faculté de médecine. Depuis peu, l'Institut 
dispose de l'ensemble de cette belle maison dans laquelle ont été aménagés une bibliothèque, une salle de lecture et de conférences, les bureaux pour les chercheurs et le personnel technique ainsi qu'un compactus en sous-sol. Ainsi, outre les chercheurs qui lui sont attachés, l'Institut peut et veut accueillir des chercheurs extérieurs auxquels il met volontiers à disposition les ressources dont il dispose.

(Ph.M.) Vous venez de mentionner la bibliothèque. Mais étant donné que l'Institut est de création récente, cette bibliothèque est-elle déjà opérationnelle? Quelles ressources offre-t-elle à l'utilisateur?

(B. F.) Elle est en voie de constitution. Nous sommes en train de la doter de l'ensemble des instruments de travail nécessaires à la recherche en histoire de la médecine tels qu'ouvrages de références et répertoires bibliographiques. Notre «jeunesse» fait que nous ne disposons évidemment pas de ce fonds d'ouvrages que les bibliothèques plus anciennes ont pu se constituer au cours des années. Mais il faut préciser que la bibliothèque de la toute proche Faculté de médecine ainsi que la Bibliothèque Publique et Universitaire de Genève possèdent beaucoup d'ouvrages concernant l'histoire de la médecine. En outre, nous sommes intégrés dans le réseau Sibil, qui nous donne accès aux catalogues informatisés des grandes bibliothèques de Suisse et nos moyens financiers nous permettent d'acquérir les nouveautés, et d'enrichir peu à peu notre fonds grâce à des dons et à l'achat de livres d'occasion. D'autre part, nous avons mis sur pied, avec l'accord de la Faculté, une politique de rapatriement d'importants fonds anciens qui se trouvent dispersés dans les différents départements et cliniques. J'ajouterai pour terminer que notre bibliothèque et nos bureaux sont dotés de moyens informatiques performants, indispensables aujourd'hui au bon fonctionnement d'un institut de recherche.

(Ph.M.) Telles sont donc les ressources matérielles de l'Institut. Mais qu'en estil des ressources humaines?

(B. F.) L'Institut Louis Jeantet dispose d'un poste de professeur qui a rang de professeur ordinaire à la Faculté de médecine. S'y ajoutent un poste de maître d'enseignement et de recherche, un poste de professeur associé (à temps partiel), un poste de privat docent, un poste de chargé de cours (à temps partiel) et un poste d'assistant actuellement partagé entre deux personnes à $50 \%$ chacune. 
Notre dotation en personnel administratif et technique se compose d'un poste de bibliothécaire et d'un poste de secrétaire.

(Ph. M.) Donnerez-vous à l'Institut Louis Jeantet d'histoire de la médecine une ligne particulière qui oriente son activité et notamment ses programmes de recherche?

(B. F.) Comme préalable à ma réponse je tiens tout d'abord à insister sur la vocation de recherche de l'Institut. Le principe qui nous guide est de nous ouvrir aux horizons les plus variés en fonction de l'orientation intellectuelle des chercheurs que nous accueillerons. Chacun gardera sa liberté dans l'orientation de ses travaux, notre ambition étant d'assurer à tous les meilleures conditions possibles sur le plan matériel comme sur le plan intellectuel. A cet égard, un accent particulier sera mis sur une politique active d'échange avec des chercheurs d'autres institutions et sur l'accueil dans notre Institut de visiting professors.

Cela dit, nous définissons quand même des centres de gravité dans l'activité de l'Institut. Il ne s'agit pas de spécialisations limitatives qui seraient en contradiction avec le principe général que je viens d'énoncer. Il s'agit bien plutôt de déterminer ce que j'appellerai des orientations d'excellence aptes à donner une personnalité propre à l'Institut Louis Jeantet. Pour l'instant, nous avons dégagé deux grands axes de recherche sur lesquels nous mettons l'accent:

1. L'histoire des idées médicales de l'Antiquité à nos jours. On peut dire que l'Institut poursuit ainsi une tradition illustrée avec éclat à Genève par l'enseignement et les travaux du professeur Jean Starobinski. Le séminaire de cette année académique 1992/93, «Médecines antiques et médecines préscientifiques», animé par Vincent Barras, maître assistant dans notre Institut, s'inscrit précisément dans cette orientation.

2. L'histoire de la collaboration sanitaire internationale, par quoi nous entendons non seulement l'histoire de l'Organisation mondiale de la santé (OMS), mais d'un point de vue plus vaste l'histoire de la santé internationale, c'est-à-dire de l'ensemble des relations entre Etats dans le domaine de la santé publique, de l'hygiène publique, des épidémies et cela depuis le Moyen Age. Nous avons déjà pris des contacts avec l'OMS, dont le siège est à Genève, et le projet a pris corps de créer à l'Institut Louis Jeantet un centre d'histoire de la médecine et de la collaboration sanitaire agréé par l'OMS. Il s'agit d'un projet nécessairement international dans lequel Genève sera le 
centre d'un réseau d'instituts travaillant dans ce domaine. Une première réalisation a déjà vu le jour à propos de la malaria: un réseau de collaboration internationale a été mis sur pied dans lequel nous sommes associés notamment avec la Rockfeller Foundation, le Wellcome Institute et l'Université de Rome «La Sapienza».

C'est dans ce cadre que l'Institut Louis Jeantet organise en octobre 1993 un colloque d'une semaine à Bellagio sous l'égide de la Fondation Rockefeller avec comme thème: «Malaria and ecosystem : an historical approach». J'assure la direction scientifique du projet qui réunira les meilleurs spécialistes de l'histoire du paludisme.

(Ph.M.) Puisque nous parlons de colloques, l'Institut Louis Jeantet a-t-il déjà à cet égard des projets précis pour ces prochaines années?

(B. F.) Notre ambition est de mettre sur pied régulièrement des colloques et des conférences réunissant des chercheurs suisses et étrangers, à l'exemple de ce que nous avons réalisé à Annecy en avril 1992 en organisant en collaboration avec la Fondation Marcel Mérieux (Lyon) et la Fondation Louis Jeantet de médecine un colloque international ayant pour thème: «Emergence de maladies infectieuses: perspectives historiques». Mais comme je l'indiquais au début de notre entretien, la mise sur pied de ces colloques ne peut se faire qu'avec l'aide de financements extérieurs à notre Institut. Nous espérons que la collaboration entre la Fondation Marcel Mérieux et la Fondation Louis Jeantet de médecine puisse continuer afin de permettre à l'Institut l'organisation de conférences internationales comme celle déjà réalisée. De façon plus générale, nous avons établi un protocole de collaboration avec la Fondation Marcel Mérieux prévoyant l'organisation régulière de réunions scientifiques au Centre des Pensières (Annecy). Dans ce cadre sera organisée sur un rythme biennal une école d'été d'histoire de la médecine dont la première verra le jour en juin 1994 et sera consacrée à une réflexion pluridisciplinaire sur les limites de la vie. Cette institution continuera d'une certaine façon l'expérience de l'école d'été d'Ischia inaugurée en 1978 par le professeur Mirko Grmek et par moi-même.

Encore en 1994, nous organiserons une série de manifestations destinées à célébrer le centenaire de la découverte du bacille de la peste par Yersin. Pour cette occasion, nous préparons en particulier la publication d'une partie de la correspondance du médecin et savant vaudois. 
Nous songeons également à de petits colloques spécialisés dont le premier aura lieu à Annecy dans le courant de 1993 et aura pour thème l'histoire de la vaccine et des politiques de vaccination.

(Ph.M.) Il reste à évoquer un aspect des activités de l'Institut Louis Jeantet que nous n'avons pas encore abordé, celui de l'enseignement, cours et séminaires. Quelles sont dans ce domaine vos prestations actuelles et vos projets?

(B. F.) Je commencerai par évoquer le cours public qui existe depuis huit ans et réunit un public fidèle. Nous le maintiendrons dans sa forme actuelle, c'est-à-dire dispensé par des professeurs extérieurs à la Faculté de médecine et souvent aussi à l'Université de Genève. Les sujets en seront très variés comme ils l'ont été par le passé où ont été traités des thèmes aussi divers que l'histoire de la psychanalyse, la médecine indienne, Galien, la médecine médiévale, etc.

Nous continuerons également à inscrire au programme de l'Institut un séminaire bimensuel destiné à un public plus spécialisé, historiens, médecins, philosophes, etc.

En ce qui concerne les étudiants de la Faculté de médecine, nous prévoyons à partir de l'année académique 1993-1994 un cours d'introduction à l'histoire de la médecine qui serait donné en première année et pourrait consister, par exemple, en douze leçons d'une heure. Pour les étudiants plus avancés, nous envisageons un cours annuel commun aux Facultés de médecine et des lettres.

Enfin nous travaillons actuellement en collaboration avec le titulaire de la chaire d'histoire et de philosophie des sciences de l'Université de Genève (Prof. Jean-Claude Pont) à un projet qui devrait aboutir à des cours communs préparant à un certificat complémentaire et un certificat de spécialisation.

(Ph.M.) Puisque nous parlons de collaboration, l'envisagez-vous aussi avec les autres instituts suisses d'histoire de la médecine et sous quelle forme?

(B. F.) Pour le moment, mes collaborateurs et moi-même avons été particulièrement occupés par l'organisation interne de l'Institut et par la mise en train de ses diverses activités spécifiques. Mais il va de soi que nous envisageons dans un avenir rapproché des initiatives communes avec un ou plusieurs des autres instituts suisses d'histoire de la médecine. A cet égard, une 
collaboration étroite avec l'Institut universitaire d'histoire de la médecine et de la santé publique de Lausanne, qui ne peut être que féconde sur le plan scientifique, s'avère d'autant plus nécessaire que la situation budgétaire est difficile. La mise en commun de certaines ressources, notamment dans le domaine de la bibliothèque, l'élaboration d'une politique concertée en matière, par exemple, d'invitation de conférenciers étrangers sont des mesures rapidement réalisables qui seraient bénéfiques pour les deux instituts.

Au chapitre de la collaboration, je n'aurai garde d'oublier le cadre favorable que constitue à cet égard la Société suisse d'histoire de la médecine et des sciences naturelles. Par les liens qu'elle crée et entretient entre ses membres, par ses publications, en particulier la revue Gesnerus, par les manifestations qu'elle organise, elle représente un atout majeur pour l'essor de notre discipline.

\section{Zum Rücktritt von Privatdozent Dr. phil. II Heinz Balmer}

Nach rund zwanzigjähriger Tätigkeit als wissenschaftlicher Mitarbeiter und als geschätzter Dozent am Zürcher Institut für Medizingeschichte erreicht H. Balmer in diesem Frühjahr die Altersgrenze; auf Ende April wird er pensioniert. Doch muss er seinen Platz - trotz grosser Raumnot - nicht sofort räumen, denn er möchte noch einige Arbeiten abschliessen und die Bildersammlung des Instituts unter konservatorischen Aspekten einer genauen Überprüfung unterziehen. Und als wichtigstes wird er seine letzten Doktoranden begleiten - es werden schliesslich über hundert Dissertationen sein, die unter seiner Leitung entstanden und grossenteils in der Reihe der Zürcher Medizingeschichtlichen Abhandlungen erschienen sind.

Alle Mitarbeiter, Gäste, Besucher, Freunde und «zugewandte Orte» des Instituts sind Heinz Balmer zutiefst dankbar für seine liebenswürdige, nimmermüde, selbstlose Bereitschaft zu Hilfe und Rat, zur Öffnung seiner schier unerschöpflichen Wissensschätze und zur Suche nach Quellen - und seien sie noch so abgelegen. Alle wünschen ihm gute Gesundheit und noch viele Jahre glücklichen Nachforschens und Findens.

B. Rüttimann, Zürich 


\title{
EUROPEAN RESEARCH CONFERENCES
}

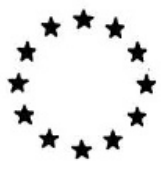

\author{
COPING WITH SICKNESS: \\ SCIENCE, CULTURE, PROFESSIONS, STATE
}

\author{
LUNTEREN (NEAR ARNHEM), THE NETHERLANDS
}

\section{5-9 SEPTEMBER 1993}

Chairman: U. Tröhler (Göttingen)

Vice-chairman: H. Beukers (Leiden)

\section{Speakers will include:}

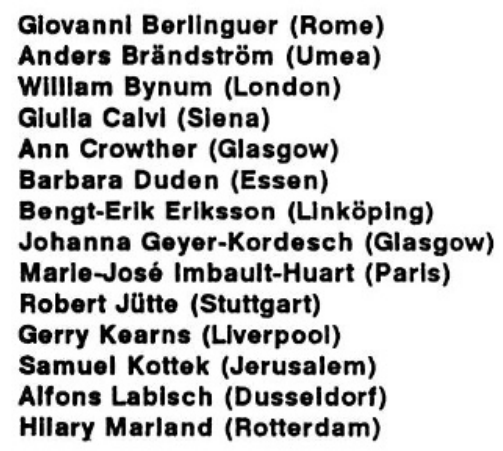

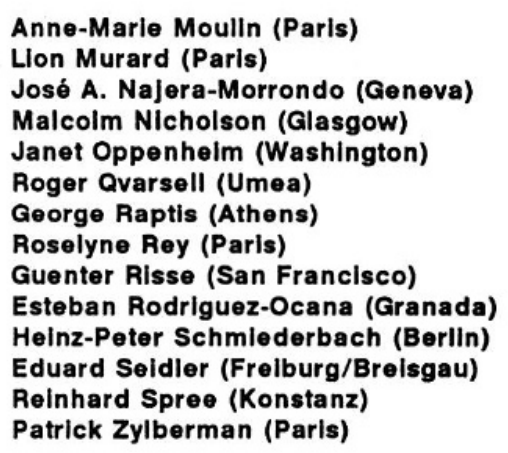

The purpose of this 1st European Research Conference on Coplng with Slckness is to promote an Interdisciplinary historical approach to medicine, mainly in Europe, trying to Integrate the different Internal and external aspects of medicine and to establish International comparisons in history. The sclentific, cultural, humanistic and social approaches to past and contemporary medicine will be emphasized In order to provide a broad basis for reflection and discussion.

The number of participants will be limited to 100 . The emphasis will be on discussion about new developments. There will be a Registration Fee covering full board and lodging. Some grants will be available for younger scientists, in particular those from less favoured regions in Europe. Deadiline for applications is 3 months before the conference.

Co-Sponsored by

the European Association for the History of Medicine and Health

For information and application forms, contact the Executive Director of the Programme:

Dr Josip Hendekovic, European Science Foundation

1 quai Lezay-Marnésia, 67080 Strasbourg Cedex, France. Tel.(33) 88767135 Fax.(33) 88366987 
The Geneva «Société de Physique et d'Histoire Naturelle» (SPHN) is pleased to announce that it is awarding a prize called:

\section{Marc-Auguste Pictet Prize}

which is meant to reward a work in the field of the History of Sciences.

The award amounts to SFr. 12000.- and can be shared.

The theme chosen for the 1994. Prize concerns:

the History of Biological Sciences

(from the Renaissance to the end of the 19th century)

Detailed information can be obtained at the following address:

Président de la SPHN

Muséum d'Histoire naturelle

Case postale 434

CH-1211 Genève 6

Suisse

Applications should be sent by 30th novembre 1993 to the above mentioned address.

\section{VI.Internationaler Leibniz-Kongress}

18.-23. Juli 1994, Hannover

Der Kongress findet in der Universität Hannover statt und beginnt am Montag, dem 18. Juli 1994, um 10 Uhr mit einer feierlichen Begrüssung und einem Festvortrag. Für Montag, den 18. Juli, 15-19 Uhr und Freitag, den 22. Juli, 15-18 Uhr sind Plenarsitzungen vorgesehen. In der übrigen Zeit des Kongresses tagen Sektionen zu verschiedenen Themenbereichen. Am Mittwoch, dem 20. Juli, um $20 \mathrm{Uhr}$ findet ein öffentlicher Vortrag statt. Während des Kongresses besteht die Möglichkeit, den Leibniz-Nachlass in der Nieder- 
sächsischen Landesbibliothek einzusehen. Samstag, der 23. Juli, bleibt Exkursionen zu Wirkungsstätten von Leibniz vorbehalten.

Die Gottfried-Wilhelm-Leibniz-Gesellschaft lädt alle interessierten Forscher, Sachkenner und Freunde zur Teilnahme ein. Anmeldungen von Sektionsbeiträgen erbitten wir bis zum 15. Dezember 1993.

Die Texte der angenommenen Beiträge werden bei Beginn des Kongresses vervielfältigt vorliegen. Die reproduzierfähigen Manuskripte (bis zu 8 Schreibmaschinenseiten) müssen spätestens am 1. März 1994 vorliegen. Genaue Angaben über die Manuskriptgestaltung werden den Referenten nach Anmeldung zugesandt.

Die Teilnehmergebühr beträgt DM 80,-. Zu den Tagungsunterlagen gehören die vervielfältigten Sektionsbeiträge.

Anfragen sind zu richten an die Gottfried-Wilhelm-Leibniz-Gesellschaft e. V., Niedersächsische Landesbibliothek, Waterloostr. 8, D-3000 Hannover 1, Telefon 0511 1267-331; Fax 0511 1267-202.

Schweizerische Gesellschaft für Geschichte der Medizin und der Naturwissenschaften

Société Suisse d'Histoire de la Médecine et des Sciences Naturelles

Vorstand 1993-1995: Präsident J. J.Dreifuss, Genève

Vizepräsident B. Rüttimann, Zürich

Sekretär/Quästor U. Boschung, Bern

Chefredaktor M. H. Bickel, Bern

Beisitzer Ph. Mudry, Lausanne

C. Müller, Bern

H. K. Schmutz, Winterthur

U.Tröhler, Göttingen/Basel

Jahrestagung der SGGMN: 24./25. September 1993

Medizinhistorisches Museum, Zürich 